\title{
New challenges for translational psychopharmacology
}

\section{Michel Bourin*}

Faculté de Médicine, Université de Nantes, Nantes Cedex, France

*Correspondence: michel.bourin@univ-nantes.fr

The young history of psychopharmacology began by studying the influence of drugs, whatever they were, on the behaviour of both rodents and human. That was the birth of psychotropic's found by serendipity. After a few years, psychopharmacology began to evaluate scientifically the "old" drugs and the new one; it was the true beginning of a new science.

Nowadays psychopharmacology is a link between the neurosciences and the psychiatry. The emergence of precise targets of drugs in the brain with the help of binding studies was a great progress in the discovery of new drugs and the beginning of the knowledge of mechanisms of action. But the main challenge is to understand the correlates on the different areas of brain and on the entire body.

Animal models for psychopathology have become an invaluable tool in the analysis of the multitude of causes, genetic, environmental or pharmacological that can bring about symptoms homologous to those of patients with a specific disorder (Bourin, 1997), despite traditional difficulties in accepting these models that stem from the argument that there is no evidence for concluding that what occurs in the brain of the animal is equivalent to what occurs in the brain of a human.

Currently animal models are sought that have three types of validity:

- Face validity, where the model is phenotypically similar and implies that the response observed in the animal model should be identical to the behavioural and physiological responses observed in humans. The behavioural responses/repertoire of mice is of course very different from the human ethogram, which includes the verbal aspect that is absent in rodents.

- Predictive validity entails that the model should be sensitive to clinically effective pharmacological agents and conversely anxiogenic compounds should elicit opposite effects, while agents that have no effect in the clinic should have no effect in these tests.

- The criterion of construct validity relates to the similarity between the theoretical rationale underlying the animal model and the human behaviour. This requires that the aetiology of the behaviour and the biological factors underlying the disorder may be similar in animals and humans.

Often researchers fail to specify if they are seeking a correlation model (e.g. predictive validity, a model that is selectivity sensitive to therapeutic agents), an isomorphic model (face validity, a model that implies the behavioural response in the human and animal is the same) or a homologous model (true construct validity, a model that implies the "cause" of the behavioural response in the animal is sufficient to provoke the same response in humans).

Behaviour can be both an event and a process and observable behaviours are the result of the integration of all of the processes ongoing in underlying organ systems, in interaction with the external social and physical environment. Animal models can allow the study of mechanisms of specific behaviours and their pathophysiology and can aid in developing and predicting therapeutic responses to pharmacological agents.

The translation of preclinical research to clinical concerns is in my opinion the main goal of psychopharmacology. For years animal models of depression, anxiety and psychosis were developed but there are mainly restricted to a core symptom of a psychiatric disorder, which is not always specific of the illness. For example, anhedonia could be part of depression as well as schizophrenia, it is the case of the chronic mild stress which itself is not a pure test predicting antidepressant activity. In the next future preclinical experimental models will gain in relevance if they incorporate maladaptive physiological or behavioural changes that have some correspondence with species behavioural adaptations. Preclinical data are more readily translated to the clinical situation when they are based on converging evidence from several experimental procedures, each capturing cardinal features of the disorder. That is the main problem to solve the gap between preclinical and clinical psychopharmacology (Shekhar et al., 2001).

Anxiety models in rodents seems to be closed to human anxiety and fear, but the pathophysiology of anxious disorders is complex, it is linked to abnormal regulation of several neurobiological components including 5-HT, GABA, glutamate, CCK during period of stress but not typically during periods of rest. We use generally ethologically based animal models of fear and anxiety attempt to approximate the natural conditions under which such emotional states are elicited. By employing non-painful aversive stimuli to induce fear and anxiety, ethological tests are thought to minimise possible confounding effects of motivational or perceptual states arising from interference with learning/memory, hunger/thirst or nociceptive mechanisms and allow for a truly comprehensive "behavioural profiling" of experimental interventions. When compared to conditioned models, ethologically based tests seem to be better qualified analogues of human anxiety. Ethological models, however, present individual differences, variable behavioural baseline levels (Bourin et al., 2007). Nonetheless, ethological stimuli are diverse in nature. Producing conditioned fear in animals requires the pairing of a previously neutral stimulus with an electric shock, excepted for the four plates test. Subsequent presentations of the stimulus disrupt ongoing behaviour and produce avoidance or defence.

In conclusion, psychopharmacology approaches will continue by ingenious innovations to make inroads in preclinical and clinical behavioural data at the interface of 
neurosciences and genetics. The challenge is to convince clinicians to study the "clinical features" of rodents at least to compare the behaviour of both humans and animals. The more closely a model approximates significant clinical symptoms, the more able it is to produce data that will induce clinical benefits (Miczek and de Wit, 2008). These new models must be validated by predicting treatments with agents having demonstrated activity in human so that leads to new drugs based on the same mechanism of action! The challenge would be to identify psychological processes pertinent to affect and cognition, to be studied in preclinical models if they are defined in behavioural and neural terms. Brain imaging also must contribute to the knowledge of drugs' mechanism of action and the location of the activity (Petit-Demoulière et al., 2009) in both animal and human. Another challenge is the modelling of bipolar disorder in rodents, to improve the discovery of mood stabilizers.

\section{REFERENCES}

Bourin, M. (1997). Animal models of anxiety: are they suitable for predicting drug action in humans? Pol. J. Pharmacol. 49, 79-84.

Bourin, M., Petit-Demoulière, B., Nic Dhonnchadha, B. A., and Hascoët, M. (2007). Animal models of anxiety in mice. Fundam. Clin. Pharmacol. 21, 567-574.

Miczek, K.A., and de Wit, H. (2008). Challenges for translational psychopharmacology research-some basic principles. Psychopharmacology 199, 291-301.

Petit-Demoulière, B., Massé, F., Cogrel, N., Hascoët, M., and Bourin, M. (2009). Brain structures implicated in the four-plate test in naîve and experienced Swiss mice using injection of diazepam and the 5-HT2A agonist DOI. Behav. Brain Res. 204, 200-205.

Shekhar, A., McCann, U. D., Meaney, M. J., Blanchard, D. C., Davis, M., Frey, K. A., Liberzon, I., Overall, K L., Shear, M. K., Tecott, L. H., and Winsky, L. (2001). Summary of a National Institute of Mental Health workshop: developing animal models of anxiety disorders. Psychopharmacology 157, 327-339.

Received: 13 January 2010; accepted: 13 January 2010; published online: 18 January 2010.

Citation: Bourin M (2010) New challenges for translational psychopharmacology. Front. Psychiatry 1:3. doi: 10.3389/ fpsyt.2010.00003

This article was submitted to Frontiers in Psychopharmacology a specialty of Frontiers in Psychiatry.

Copyright (c) 2010 Bourin. This is an open-access article subject to an exclusive license agreement between the authors and the Frontiers Research Foundation, which permits unrestricted use, distribution, and reproduction in any medium, provided the original authors and source are credited. 